\title{
Combined Intravitreal Dexamethasone Implant and Cataract Surgery in Patients with Diabetic Retinopathy: Effect on Retinal Morphology and Function
}

\author{
Angelo Maria Minnella · Martina Maceroni (D) - Stefano Maria Picardi · \\ Giorgio Placidi · Elisa De Siena $\cdot$ Stanislao Rizzo $\cdot$ Benedetto Falsini
}

Received: August 16, 2020 / Accepted: September 14, 2020 / Published online: September 24, 2020

(C) The Author(s) 2020

\section{ABSTRACT}

Introduction: Cataract surgery can be associated with vision-threatening complications in patients with diabetes. This study aimed to assess the functional and anatomic outcomes of the intravitreal dexamethasone (DEX) implant, administered at the time as cataract surgery, in patients with diabetic retinopathy and diabetic macular edema (DME).

Methods: This was a retrospective, observational, and single-center study. The primary endpoint was the mean change in central macular thickness (CMT) from baseline to month 1 . Secondary endpoints included mean change in best corrected visual acuity (BCVA) from baseline to month 1 and 3, mean change in CMT from baseline to month 3, the photopic negative response (PhNR) and the $b$ wave of flash full-field electroretinogram from baseline

Digital Features To view digital features for this article go to https://doi.org/10.6084/m9.figshare.12944189.

A. M. Minnella · M. Maceroni $(\square)$.

S. M. Picardi · G. Placidi · E. De Siena · S. Rizzo ·

B. Falsini

Università Cattolica del Sacro Cuore, Rome, Italy

e-mail: maceronimartina@gmail.com

A. M. Minnella - M. Maceroni - S. M. Picardi ·

G. Placidi · E. De Siena - S. Rizzo · B. Falsini

UOC Oftalmologia, Fondazione Policlinico

Universitario A. Gemelli, IRCCS, Rome, Italy to month 1, and the incidence of adverse events.

Results: Twenty-four eyes of 21 patients were included in the study. The mean (range) age of patients was 69 (63-87) years and 13 (61.9\%) were men. Mean (standard deviation) CMT significantly decreased from 447 (134) $\mu \mathrm{m}$ at baseline to 341 (134) $\mu \mathrm{m}$ at month 1 (mean difference $-106 \pm 134 \mu \mathrm{m}, 95 \% \mathrm{CI}-183.9$ to $-28.1 \mu \mathrm{m} ; \quad p=0.0087)$. BCVA significantly improved from 46 (20) ETDRS letters at baseline to 59 (22) ETDRS letters at month 1 (mean difference $13 \pm 21$ letters, 95\% CI 0.8-25.2 letters; $p=0.0375)$. Regarding electrophysiology, there was a statistically significant reduction in mean PhNR from $5.24(1.67) \mu \mathrm{V}$ at baseline to 3.73 (1.19) $\mu \mathrm{V}$ at month 1 (mean difference $-1.51 \pm 0.42 \mu \mathrm{V}, 95 \% \mathrm{CI}-2.4$ to $-0.7 \mu \mathrm{V}$, $p=0.0008)$; whereas $\mathrm{b}$ wave amplitude did not change $(12.69 \pm 6.89 \mu \mathrm{V}$ at baseline versus $12.29 \pm 6.30 \mu \mathrm{V}$ at month $1 ; p=0.8347)$. Four (16.7\%) eyes developed ocular hypertension over the course of follow-up, which was successfully controlled with topical hypotensive medication.

Conclusion: Perioperative DEX implant significantly improved both anatomic and functional outcomes in patients with DME who underwent cataract surgery.

Keywords: Cataract; Diabetes; Diabetic macular edema; Diabetic retinopathy; 
Electrophysiology; Macular thickness; Personalized medicine; Visual acuity

\section{Key Summary Points}

Why carry out this study?

Compared to patients without diabetes, cataract surgery outcomes were reported to be worse in patients with diabetes, especially in those with diabetic retinopathy. A preexisting diabetic macular edema could increase the risk of ME progression by $20-50 \%$; hence, an appropriate therapeutic management of DME is recommended perioperatively.

This study aimed to assess the functional and anatomic outcomes of the intravitreal dexamethasone implant, administered at the time as cataract surgery, in patients with diabetic retinopathy and diabetic macular edema.

\section{What was learnt from the study?}

In patients with diabetes who underwent cataract surgery, perioperative DEX implant significantly improved both anatomic and functional outcomes and may effectively prevent the diabetic macular edema worsening associated with cataract surgery.

It is therefore crucial to identify patients with diabetes prior to cataract surgery so that they can benefit from adequate therapeutic preventive measures to reduce the risk of postoperative complications.

\section{DIGITAL FEATURES}

This article is published with digital features to facilitate understanding of the article. To view digital features for this article go to https://doi. org/10.6084/m9.figshare.12944189.

\section{INTRODUCTION}

Diabetic macular edema (DME) is the leading cause of vision loss in patients with diabetic retinopathy (DR) [1]. According to the Wisconsin Epidemiologic Study of Diabetic Retinopathy (WESDR), the 10-year incidence of DME was $20 \%$ for patients with type 1 diabetes mellitus (DM), $13.9 \%$ for patients wtih type 2 diabetes receiving insulin, and $25.4 \%$ for patients with non-insulin-dependent type 2 DM [1].

Patients with DME are more likely to develop cataract because of the inherent metabolic condition [2]; therefore, a large percentage of patients with diabetes undergoes cataract surgery every year. According to a recent study, $20.4 \%$ of patients undergoing cataract surgery in Italy are diabetic [3]. Cataract surgery can be associated with vision-threatening complications in patients with diabetes, such as DME, postoperative macular edema, progression of DR, and posterior capsular opacification [4].

Compared to patients without diabetes, cataract surgery outcomes were reported to be worse in patients with diabetes, especially in those with DR $[5,6]$. As preexisting DME can increase the risk of macular edema (ME) progression by $20-50 \%$, an appropriate therapeutic management of DME is recommended perioperatively [7].

Surgical inflammation associated with cataract surgery may be responsible for poor functional outcomes in patients with DME [4].

Since there is increasing evidence about the role of inflammation in the pathophysiology of DME, corticosteroids have taken an active role in its treatment [8]. Corticosteroid therapy is able to inhibit many of the processes known to be involved in the progression of DME, through anti-inflammatory properties [9] and vascular endothelial growth factor (VEGF) inhibition [10]. Therefore, the perioperative administration of a dexamethasone intravitreal (DEX) implant in patients with diabetes undergoing cataract surgery might be beneficial.

We have evidence suggesting that, in patients with diabetes, the intraoperative use of 
a DEX implant in combination with phacoemulsification and intraocular lens (IOL) implantation could provide good functional and anatomic outcomes [11-14]. It has been clearly demonstrated that DR affects not only retinal vasculature but also the neural elements of the retina; in fact, retinal ganglion cells (RGCs) are particularly susceptible to glutamate excitotoxicity, which plays an important role in ischemic diseases such as vessel occlusion and DR [15].

Electroretinography can estimate the overall functional status of the retina. The photopic negative response (PhNR) of flash full-field electroretinogram (ERG) is the negative potential which follows the $b$ wave. It is an electrical signal that represents the activity of RGCs and their axons. It was suggested that PhNR may be useful for assessing the inner retinal damage and how it progressively decreases with the progression of DR [16]. Furthermore, reduced b wave amplitudes and prolonged implicit times, for rod- and cone-driven responses, are related to retinal impairment in DR [17].

Since inflammation and altered concentrations of angiogenic factors after cataract surgery may aggravate maculopathy, we hypothesized that perioperative use of DEX implant may be effective for reducing the potential DME worsening after phacoemulsification. Additionally, the assessment of retinal and visual function in patients with diabetic retinopathy and cataract, as well as the prediction of the recovery of the visual acuity of patients after cataract surgery, could be relatively difficult. Electrophysiological examination has therefore been used as an objective examination technique for visual function.

The first purpose of this study was to evaluate the anatomic response of DEX implant administered at the time as cataract surgery in patients with DR, with DME, and central macular thickness (CMT) greater than $250 \mu \mathrm{m}$. Additionally, this study aimed to evaluate the functional effect of DEX implant, in terms of best corrected visual acuity (BCVA) and electrophysiological parameters (amplitude of b wave and PhNR), and safety, evaluating intraocular pressure (IOP) variations and incidence of adverse events.

\section{METHODS}

The CATOZURDEX study was designed as a retrospective, observational, and single-center study conducted at the Ophthalmology Unit of Università Cattolica del S. Cuore/Fondazione Policlinico Universitario A. Gemelli-IRCCS of Rome, which included consecutive adult (over 18 years old) patients with DR and DME who underwent cataract surgery and an intraoperative DEX implant, between January 2017 and December 2019. Patients with CMT equal or greater than $250 \mu \mathrm{m}$, as measured using an optical coherence tomography (OCT) device, were suitable to be included in the study.

This study adhered to the tenets of the Declaration of Helsinki and was approved by the ethics committee of Fondazione Policlinico Universitario A. Gemelli, which waived the need for informed consent for this retrospective study.

Patients with a history of glaucoma, high intraocular pressure (defined as IOP $>21 \mathrm{mmHg}$ ), and patients with retinal or choroidal disease other than DR that could affect CMT were excluded. Dense retinal exudates and media opacity that could interfere with the CMT analysis were considered additional exclusion criteria.

All eyes underwent phacoemulsification (Alcon Centurion Vision System; Forth Worth, TX, USA) and "in the bag" lens implantation (Alcon Acrysof SA60AT) by a single surgeon (AMM) under topical anesthesia. At the end of the surgical procedure, DEX implant $\left(\right.$ Ozurdex $^{\mathrm{TM}}$, Allergan plc., Dublin, Ireland) was injected in the inferotemporal quadrant at 3.5-4 $\mathrm{mm}$ from scleracorneal limbus. DEX implant was injected at the end of the phacoemulsification procedure, when potential intraoperative complications had been overcome.

Postoperative care included antibiotic (moxifloxacin) four times daily for 2 weeks; a topical non-steroidal anti-inflammatory (nepafenac 3\%) three times daily for 2 weeks and then once daily for 2 months; and a topical steroid (dexamethasone $0.15 \%$ ) four times a day, which was slowly tapered over 4 weeks. 
The protocol included a pre-surgery visit that was considered as baseline visit (performed the day before surgery) and three follow-up visits. Follow-up visits were considered at week 1 ( \pm 2 days) and months 1 and 3 ( \pm 2 weeks).

All patients underwent a complete ophthalmologic examination. Slit lamp biomicroscopy, BCVA, dilated fundus exam, and IOP measurement were performed at each visit and spectral domain OCT at baseline, month 1 , and month 3; OCT analysis was performed using Heidelberg Spectralis Spectral Domain OCT (Heidelberg Engineering, Heidelberg, Germany). CMT was manually assessed on horizontal B scans. Early Treatment Diabetic Retinopathy Study (ETDRS) charts were used to assess BCVA, while IOP was measured by means a Goldmann applanation tonometer.

DME recurrence was defined as worsening in visual acuity of at least 5 letters and/or a CMT thickening of at least $50 \mu \mathrm{m}$, as compared to the best postoperative values reached at 1 month.

\section{Electrophysiology}

Flash full-field ERG and PhNR were performed at baseline and at month 1 after phacoemulsification.

For each patient, Ganzfeld electroretinograms were recorded with a specific, published protocol employed to isolate and analyze the PhNR from the single flash cone-mediated responses [18-20]. The amplitudes of the PhNR and of the cone $b$ wave were measured in each recording session using a Retimax instrument (CSO Company Florence, Italy).

\section{Outcome Measures}

The primary endpoint was the mean change in CMT from baseline to month 1. Secondary endpoints included mean change in CMT at 3 months, mean change in BCVA from baseline to months 1 and 3, ERG parameters (PhNR and $\mathrm{b}$ wave) from baseline to month 1 , and incidence of adverse events.

\section{Statistical Analysis}

Before starting the study, it was determined that a sample size of 22 patients provided an $85 \%$ power, at an alpha of 0.05 , to detected a difference of $25 \%$ in PhNR and b wave amplitude after treatment compared to baseline values.

Descriptive statistics number (percentage), mean [standard deviation (SD)], mean [95\% confidence interval $(95 \% \mathrm{CI})]$, mean [standard error (SE)], or median (95\% CI) were used, as appropriate.

Data were tested for normal distribution using the normality test (Shapiro-Wilk test). As data were normally distributed, the two-way paired-sample $t$ test was used to compare means at baseline and month 1 .

Categorical variables were compared using a chi-square test and a Fisher's exact test, as appropriated.

\section{RESULTS}

Among the 43 screened patients, 21 (24 eyes) met all the requirements of the inclusion/exclusion criteria. The mean (range) age of patients was 69 (63-87) years and 13 (61.9\%) patients were men. The main baseline demographic and clinical characteristics of the study sample are summarized in Table 1.

Mean (standard deviation) CMT significantly decreased from 447 (134) $\mu \mathrm{m}$ at baseline to 341 (134) $\mu \mathrm{m}$ at month 1 (mean difference $-106 \pm 134 \mu \mathrm{m}, 95 \% \mathrm{CI}-183.9$ to $-28.1 \mu \mathrm{m}$; $p=0.0087)$. Seventeen (70.8\%) eyes achieved a CMT reduction of at least 20\%. Data were plotted from CMT baseline on the $\mathrm{x}$-axis and month 1 CMT on the y-axis to make an overall visual assessment, and the data were not distributed around the line (Fig. 1).

At month 3, CMT reduction remained stable in $22(91.7 \%)$ eyes, while two $(8.3 \%)$ showed a CMT worsening (CMT thickening of at least $50 \mu \mathrm{m}$ as compared to month 1).

BCVA significantly improved from 46 (20) ETDRS letters at baseline to 59 (22) ETDRS let- 
Table 1 Baseline clinical and demographic characteristics of the study sample

\begin{tabular}{|c|c|}
\hline Variable & $N=\mathbf{2 4}$ \\
\hline \multicolumn{2}{|l|}{ Age, years* } \\
\hline Mean & 69 \\
\hline Range & $63-87$ \\
\hline \multicolumn{2}{|l|}{ Sex* ${ }^{*}, n(\%)$} \\
\hline Female & $8(38.1)$ \\
\hline Male & $13(61.9)$ \\
\hline \multicolumn{2}{|l|}{ Eye, $n(\%)$} \\
\hline Right & $16(66.7)$ \\
\hline Left & $8(33.3)$ \\
\hline \multicolumn{2}{|c|}{ Type of $\mathrm{DM}^{*}, n(\%)$} \\
\hline Type 1 & $0(0.0)$ \\
\hline Type 2 & $21(100.0)$ \\
\hline \multicolumn{2}{|c|}{ DM treatment*, $n(\%)$} \\
\hline Insulin & $13(61.9)$ \\
\hline $\mathrm{OAD}$ & $8(38.1)$ \\
\hline \multicolumn{2}{|c|}{ Diabetic retinopathy*, $n(\%)$} \\
\hline No-proliferative & $9(42.9)$ \\
\hline Proliferative & $12(57.1)$ \\
\hline \multicolumn{2}{|c|}{ Length of DM, years } \\
\hline Mean & 5.3 \\
\hline Range & $2-35$ \\
\hline \multicolumn{2}{|l|}{$\mathrm{CMT}, \mu \mathrm{m}$} \\
\hline Mean (SD) & $447(134)$ \\
\hline \multicolumn{2}{|l|}{ BCVA, letters* ${ }^{*}$} \\
\hline Mean (SD) & $46(20)$ \\
\hline \multicolumn{2}{|l|}{ PhNR, $\mu \mathrm{V}$} \\
\hline Mean (SD) & $5.24(1.67)$ \\
\hline $\mathrm{B}$ wave, $\mu \mathrm{V}$ & \\
\hline
\end{tabular}

Table 1 continued

\begin{tabular}{ll}
\hline Variable & $\boldsymbol{N}=\mathbf{2 4}$ \\
\hline Mean $(\mathrm{SD})$ & $12.69(6.89)$ \\
\hline
\end{tabular}

$N$ number, $S D$ standard deviation, $C I$ confidence interval, $D M$ diabetes mellitus, $O A D$ oral antidiabetic drugs, $C M T$ central macular thickness, $B C V A$ best corrected visual acuity, $P h N R$ photopic negative response

${ }^{*}$ By patient (a total of 21 patients)

${ }^{* *}$ Letters in the Early Treatment Diabetic Retinopathy Study (ETDRS) charts

ters at month 1 (mean difference $13 \pm 21$ letters, 95\% CI $0.8-25.2$ letters; $p=0.0375$ ) (Fig. 2). Eleven (45.8\%) eyes achieved a mean BCVA improvement of at least 15 ETDRS letters, $5(20.8 \%)$ eyes reached a mean BCVA improvement of at least 5 letters, and 8 (33.3\%) eyes achieved a mean BCVA improvement of less than 5 letters. At month 3, mean BCVA was 55 (range 2-85) letters, with 23 (95.8\%) eyes remaining stables and only $1(4.2 \%)$ showing visual acuity deterioration (visual acuity worsening of at least 5 letters as compared to month 1).

Regarding electrophysiology, there was a statistically significant reduction in mean PhNR from 5.24 (1.67) $\mu \mathrm{V}$ at baseline to 3.73 (1.19) $\mu \mathrm{V}$ at month 1 (mean difference $-1.51 \pm 0.42 \mu \mathrm{V}$, 95\% CI -2.4 to $-0.7 \mu \mathrm{V}, p=0.0008$ ) (Fig. 3); whereas b wave amplitude did not change $(12.69 \pm 6.89 \mu \mathrm{V}$ at baseline versus $12.29 \pm 6.30 \mu \mathrm{V}$ at month 1 ; mean difference - $0.40 \pm 1.91 \mu \mathrm{V}, 95 \% \mathrm{CI}-4.24$ to $3.44 \mu \mathrm{V}$, $p=0.8347$ ) (Fig. 4).

Regarding safety, 4 (16.7\%) eyes developed ocular hypertension over the course of followup; this hypertension was successfully controlled with topical hypotensive medication in all the subjects.

During the study other treatment-related adverse events have not been observed. 


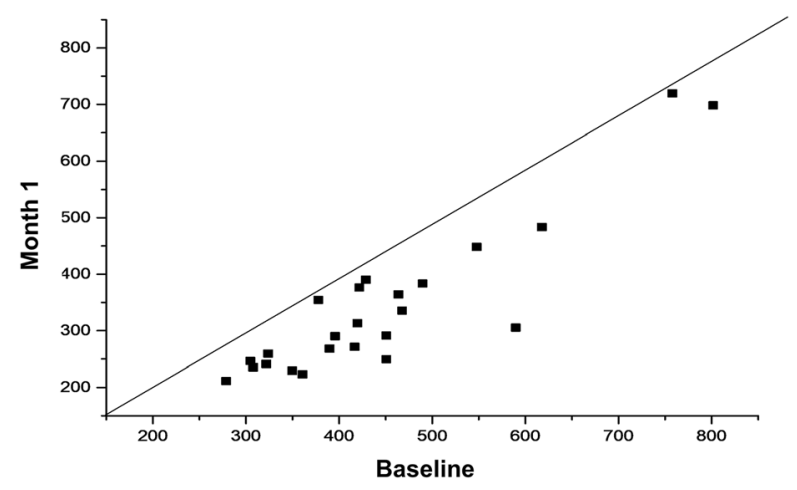

Fig. 1 Scatter plot of the baseline and month 1 central macular thickness. Mean difference $-106 \pm 134 \mu \mathrm{m}$, 95\% CI -183.9 to $-28.1 \mu \mathrm{m} ; p=0.0087$ (two-tailed paired-samples Student $t$ test)

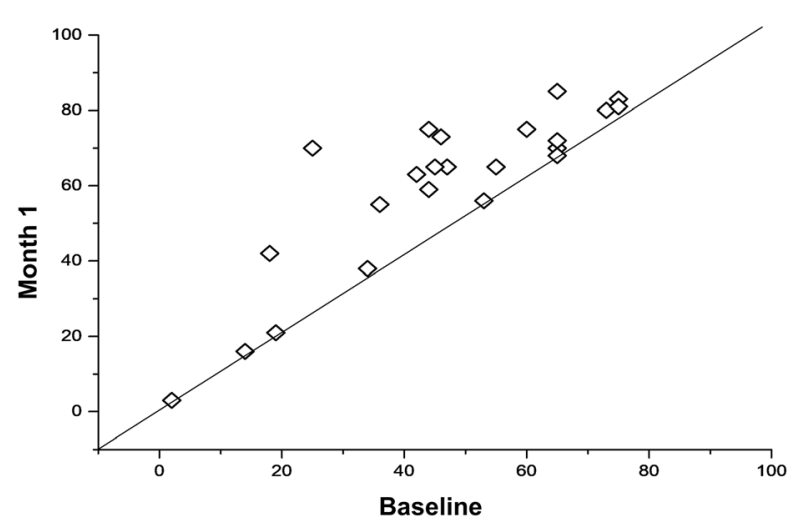

Fig. 2 Scatter plot of the baseline and month 1 best corrected visual acuity. Mean difference $13 \pm 21$ letters, 95\% CI 0.8-25.2 letters; $p=0.0375$ (two-tailed pairedsamples Student $t$ test)

\section{DISCUSSION}

The results of the current study have shown that both CMT and visual acuity outcomes significantly improved in those patients with DME who underwent a unique DEX implant at the same time as cataract surgery. By contrast the component PhNR of the Ganzfeld electroretinogram selectively decreased after treatment, while the $b$ wave remained unaltered.

Phacoemulsification in patients with diabetes and DME is associated with a high

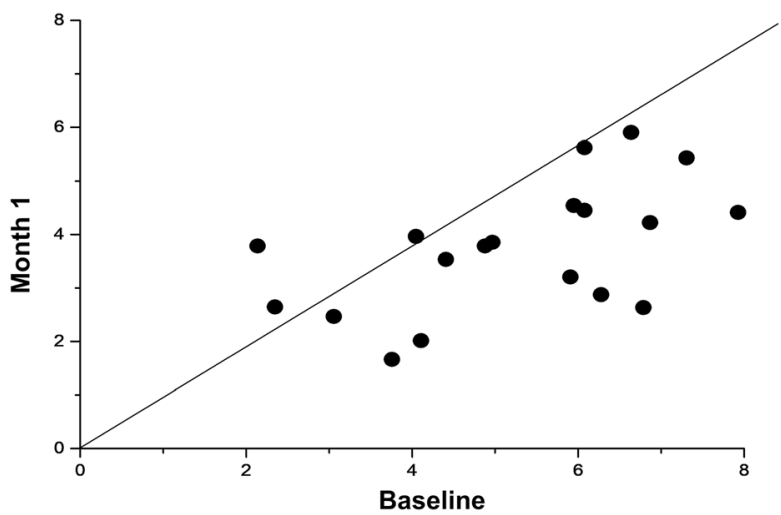

Fig. 3 Scatter plot of the photopic negative response at baseline and month 1 . Mean difference $-1.51 \pm 0.42 \mu \mathrm{V}, 95 \% \mathrm{CI}-2.4$ to $-0.7 \mu \mathrm{V}$; $p=0.0008$ (two-tailed paired-samples Student $t$ test)

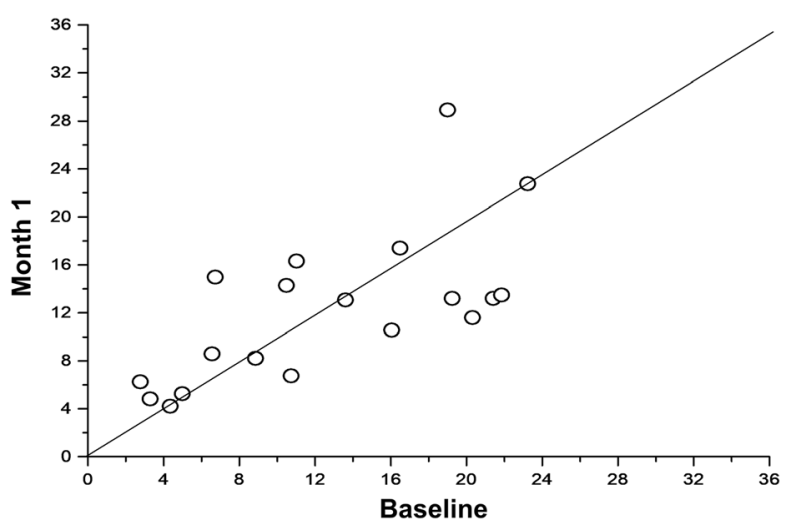

Fig. 4 Scatter plot of the $b$ wave at baseline and month 1 . The $b$ wave amplitudes clustered around the diagonal line indicate that there were no statistically significant differences between baseline and month 1 measurements. Mean difference $-0.40 \pm 1.91 \mu \mathrm{V}, 95 \% \mathrm{CI}-4.24$ to $3.44 \mu \mathrm{V}$, $p=0.8347$ (two-tailed paired-samples Student $t$ test)

incidence of CMT worsening and with poor visual outcomes [21]. Moreover, a preexisting DME represents the main risk factor for ME worsening after uneventful cataract surgery. In fact, the incidence of pseudophakic cystoid macular edema (PCME) is significantly higher $(16.3 \%)$ in patients with previous DME and in those with DR whose blood-retinal barrier has 
been compromised before surgery [22]. Additionally, the RELDEX study, a 3-year real-life study, found that cataract surgery did not have any negative impact in those patients with DME who were treated with DEX implant [23].

Our results demonstrating better clinical outcomes in eyes that underwent a perioperative DEX implant during phacoemulsification were in line with the current literature. These findings clearly suggested that the intraoperative use of a DEX implant in combination with phacoemulsification and IOL implantation was associated with good functional and anatomic outcomes in patients with diabetes [11-14].

The peak CMT reduction was achieved at month 1 and remained stable throughout the study. Additionally, there was a significant improvement in BCVA.

As far as we know, DEX implant is currently the only intravitreal treatment for DME that can be used perioperatively, considering that anti-VEGF therapy, according to the manufacturers' summary of product characteristics, cannot be used before 1 month after surgery [24-26].

Our ERG data suggested a subclinical and temporary dysfunction of the inner retina, which was not reflected by visual acuity, after DEX implant injection. In fact, it should be noted that, as compared to baseline, PhNR decreased at month 1 in $87.5 \%(21 / 24)$ of the eyes. The $\mathrm{b}$ wave was unaltered following DEX implant.

The $\mathrm{b}$ wave is the major component of the human ERG recording. It is used in clinical and experimental analysis as an indicator of retinal function. It originates in retinal cells that are post-synaptic to the photoreceptors [27]. Because the $b$ wave reflects the sum of the negative P-III component and the positive P-II component, its amplitude is measured from the trough of the a wave to the peak of the $b$ wave [27].

On the other hand, the PhNR is represented by the negative wave following the $b$ wave. It originates from the RGC and their axons. As compared to healthy subjects, the PhNR amplitude was reduced and the implicit time was prolonged in patients with DR [28]. RGCs are particularly vulnerable to glutamate- induced cellular toxicity. Therefore, increased glutamate in the vitreous of patients with DR induced degeneration of retinal tissue cells, which might play an important role in retinal ischemia [15].

Kizawa et al. reported that the $\mathrm{PhNR} / \mathrm{b}$ wave amplitude ratio was normal in patients with DR, but the ratio was significantly reduced as the DR became more severe [29]. These results suggested that RGCs suffered a more severe damage from diabetic changes than the cone cells or bipolar cells did [28].

In healthy subjects, the amplitude of the b wave was closely correlated with postoperative BCVA for the scotopic 3.0 ERG response [30].

Similarly, in patients with diabetes, we have evidence suggesting a significant reduction of multifocal ERG components and a delay in their latencies in eyes with DME, which clearly indicates a functional impairment in the outer retina. Moreover, there was a significant inverse association between BCVA and the amplitude of $\mathrm{P} 1$ and N2, and the latency of central N1. However, this study failed to find any correlation between ERG parameters and CMT [31].

This study has some limitations that should be taken into consideration when interpreting its results. The first one is its design. Although retrospective designs provide a valid vehicle for research, they can be riddled with threats to both internal and external validity. To minimize this issue, this study applied strict inclusion/exclusion criteria. The second limitation is the restricted number of patients that can be included in a single-center study. Nevertheless, prior to the study, calculation of the sample size was performed. Another limitation is the fact that ERG may be affected by different factors, e.g., temperature of the vitreous, light intensity, state of adaptation, etc. [27].

\section{CONCLUSION}

In patients with DME who underwent cataract surgery, perioperative DEX implant significantly improved both anatomic and functional outcomes. Additionally, DEX may effectively prevent the DME worsening associated with 
cataract surgery. In order to reduce the risk of postoperative complications, it is therefore crucial to identify patients with diabetes prior to cataract surgery so that they can benefit from adequate therapeutic preventive measures. Further studies are needed to elucidate the relationship between the ERG components and the BCVA and the retinal thickness.

\section{ACKNOWLEDGEMENTS}

Funding. No funding or sponsorship was received for this study. Allergan SA provided funding for the journal's Rapid Service fee and Open Access publication.

Editorial Assistance. Editorial assistance in the preparation of this article was provided by Dr. Antonio Martínez of Ciencia y Deporte Ltd. Support for this assistance was funded by Allergan SA.

Authorship. All named authors meet the International Committee of Medical Journal Editors (ICMJE) criteria for authorship for this article, take responsibility for the integrity of the work as a whole, and have given their approval for this version to be published.

Disclosures. Prof Angelo Maria Minnella has received a Grant from Allergan during the conduct of the study. Martina Maceroni, Stefano Maria Picardi, Giorgio Placidi, Elisa De Siena, Stanislao Rizzo and Benedetto Falsini have nothing to disclose.

Compliance with Ethics Guidelines. All procedures performed in studies involving human participants were in accordance with the ethical standards of the institutional and/or national research committee and with the 1964 Helsinki declaration and its later amendments or comparable ethical standards. The study was approved by the ethics committee of Fondazione Policlinico Universitario A. Gemelli (protocol ID: 3308), which waived the need for informed consent for this study.
Data Availability. The datasets generated during and/or analyzed during the current study are available from the corresponding author on reasonable request.

Open Access. This article is licensed under a Creative Commons Attribution-NonCommercial 4.0 International License, which permits any non-commercial use, sharing, adaptation, distribution and reproduction in any medium or format, as long as you give appropriate credit to the original author(s) and the source, provide a link to the Creative Commons licence, and indicate if changes were made. The images or other third party material in this article are included in the article's Creative Commons licence, unless indicated otherwise in a credit line to the material. If material is not included in the article's Creative Commons licence and your intended use is not permitted by statutory regulation or exceeds the permitted use, you will need to obtain permission directly from the copyright holder. To view a copy of this licence, visit http://creativecommons.org/licenses/by$\mathrm{nc} / 4.0 /$.

\section{REFERENCES}

1. Klein R, Klein BE, Moss SE, Cruickshanks KJ. The Wisconsin epidemiologic study of diabetic retinopathy. XV. The long-term incidence of macular edema. Ophthalmology. 1995;102:7-16.

2. Sayin N, Kara N, Pekel G. Ocular complications of diabetes mellitus. World J Diabetes. 2015;6(1): 92-108.

3. Panozzo G, Staurenghi G, Dalla Mura G, et al. Prevalence of diabetes and diabetic macular edema in patients undergoing senile cataract surgery in Italy: the diabetes and CATaract study. Eur J Ophthalmol. 2019;30(2):315-20.

4. Peterson SR, Silva PA, Murtha TJ, Sun JK. Cataract surgery in patients with diabetes: management strategies. Semin Ophthalmol. 2018;33(1):75-82. https://doi.org/10.1080/08820538.2017.1353817.

5. Krepler K, Biowski R, Schrey S, Jandrasits K, Wedrich A. Cataract surgery in patients with diabetic retinopathy: visual outcome, progression of diabetic retinopathy, and incidence of diabetic 
macular oedema. Graefes Arch Clin Exp Ophthalmol. 2002;240(9):735-8.

6. Eriksson U, Alm A, Bjärnhall G, Granstam E, Matsson AW. Macular edema and visual outcome following cataract surgery in patients with diabetic retinopathy and controls. Graefes Arch Clin Exp Ophthalmol. 2011;249(3):349-59.

7. Diabetic Retinopathy Clinical Research Network, Baker CW, Almukhtar T, et al. Macular edema after cataract surgery in eyes without preoperative central-involved diabetic macular edema. JAMA Ophthalmol. 2013;131(7):870-9. https://doi.org/10. 1001/jamaophthalmol.2013.

8. Al Dhibi HA, Arevalo JF. Clinical trials on corticosteroids for diabetic macular edema. World J Diabetes. 2013;4(6):295-302. https://doi.org/10.4239/ wjd.v4.i6.295.

9. Sohn HJ, Han DH, Kim IT, et al. Changes in aqueous concentrations of various cytokines after intravitreal triamcinolone versus bevacizumab for diabetic macular edema. Am J Ophthalmol. 2011;152(4):686-94. https://doi.org/10.1016/j.ajo. 2011.03.033.

10. Brooks HL Jr, Caballero S Jr, Newell CK, et al. Vitreous levels of vascular endothelial growth factor and stromal-derived factor 1 in patients with diabetic retinopathy and cystoid macular edema before and after intraocular injection of triamcinolone. Arch Ophthalmol. 2004;122(12):1801-7.

11. Agarwal A, Gupta V, Ram J, Gupta A. Dexamethasone intravitreal implant during phacoemulsification. Ophthalmology. 2013;120(1):211.e1-5.

12. Panozzo GA, Gusson E, Panozzo G, Dalla MG. Dexamethasone intravitreal implant at the time of cataract surgery in eyes with diabetic macular edema. Eur J Ophthalmol. 2017;27(4):433-7. https://doi.org/10.5301/ejo.5000920.

13. Furino C, Boscia F, Niro A, et al. Combined phacoemulsification and intravitreal dexamethasone implant (Ozurdex $\left.{ }^{\circledR}\right)$ in diabetic patients with coexisting cataract and diabetic macular edema. J Ophthalmol. 2017;2017:4896036. https://doi.org/ $10.1155 / 2017 / 4896036$.

14. Calvo P, Ferreras A, Al Adel F, Dangboon W, Brent $\mathrm{MH}$. Effect of an intravitreal dexamethasone implant on diabetic macular edema after cataract surgery. Retina. 2018;38(3):490-6. https://doi.org/ 10.1097/IAE.0000000000001552.

15. Nakazawa T, Takahashi $H$, Nishijima $K$, et al. Pitavastatin prevents NMDA-induced retinal ganglion cell death by suppressing leukocyte recruitment. J Neurochem. 2007;100(4):1018-31.
16. Chen H, Zhang M, Huang S, Wu D. The photopic negative response of flash ERG in nonproliferative diabetic retinopathy. Doc Ophthalmol. 2008;117(2):129-35.

17. Dimopoulos IS, Freund PR, Redel T, Dornstauder B, Gilmour G, Sauvé Y. Changes in rod and cone-driven oscillatory potentials in the aging human retina. Invest Ophthalmol Vis Sci. 2014;55(8): 5058-73.

18. Abed E, Piccardi M, Rizzo D, et al. Functional loss of the inner retina in childhood optic gliomas detected by photopic negative response. Invest Ophthalmol Vis Sci. 2015;56(4):2469-74.

19. Falsini B, Chiaretti A, Rizzo D, et al. Nerve growth factor improves visual loss in childhood optic gliomas: a randomized, double-blind, phase II clinical trial. Brain. 2016;139(Pt 2):404-14.

20. Abed E, Placidi G, Campagna F, et al. Early impairment of the full-field photopic negative response in patients with Stargardt disease and pathogenic variants of the ABCA4 gene. Clin Exp Ophthalmol. 2018;46(5):519-30.

21. Squirrell D, Bhola R, Bush J, Winder S, Talbot JF. A prospective, case controlled study of the natural history of diabetic retinopathy and maculopathy after uncomplicated phacoemulsification cataract surgery in patients with type 2 diabetes. Br J Ophthalmol. 2002;86(5):565-71.

22. Munk MR, Jampol LM, Simader C, et al. Differentiation of diabetic macular edema from pseudophakic cystoid macular edema by spectraldomain optical coherence tomography. Invest Ophthalmol Vis Sci. 2015;56:6724-33.

23. Malclès A, Dot C, Voirin N, et al. Real-life study in diabetic macular edema treated with dexamethasone implant: the Reldex study. Retina. 2017;37(4): 753-60.

24. Avastin ${ }^{\mathrm{TM}}$. Safety data sheet. 2020. https://www. gene.com/download/pdf/AvastinVials400mgSDS3. pdf. Accessed date 10 Aug 2020.

25. Lucentis ${ }^{\mathrm{TM}}$. Highlights of prescribing information. 2020. https://www.accessdata.fda.gov/drugsatfda docs/label/2014/125156s105lbl.pdf. Accessed $1 \overline{0}$ Aug 2020.

26. Eylea $^{\mathrm{TM}}$. Summary of product characteristics. 2020. https://www.ema.europa.eu/en/documents/ product-information/eylea-epar-productinformation_en.pdf. Accessed 10 Aug 2020.

27. Kolb H, Fernandez E, Nelson R, editors. Webvision: the organization of the retina and visual system 
[Internet]. Salt Lake City: University of Utah Health Sciences Center; 1995.

28. Kim HD, Park JY, Ohn YH. Clinical applications of photopic negative response (PhNR) for the treatment of glaucoma and diabetic retinopathy. Korean J Ophthalmol. 2010;24(2):89-95.

29. Kizawa J, Machida S, Kobayashi T, Gotoh Y, Kurosaka D. Changes of oscillatory potentials and photopic negative response in patients with early diabetic retinopathy. Jpn J Ophthalmol. 2006;50(4):367-73.
30. An J, Zhang L, Wang Y, Zhang Z. The success of cataract surgery and the preoperative measurement of retinal function by electrophysiological techniques. J Ophthalmol 2015;2015:401281.

31. Tehrani NM, Riazi-Esfahani $H$, Jafarzadehpur E, et al. Multifocal electroretinogram in diabetic macular edema; correlation with visual acuity and optical coherence tomography. J Ophthalmic Vis Res. 2015;10(2):165-71. 